\title{
Editorial
}

\section{Selected Papers from the 9th Conference of the European Association for the Teaching of Academic Writing, Royal Holloway, University of London, UK, June 2017}

The 9th conference of the European Association for the Teaching of Academic Writing (EATAW) was held in subtropical conditions from 19th - 21st June 2017 in Egham, UK. More than 400 participants from over 40 countries gathered at Royal Holloway, University of London to deliberate 'what teachers of academic writing can offer the global academy in terms of imaginative, creative and principled responses to the increasingly international, diverse and marketised reality of higher education' (EATAW 2017). As two of the co-organisers of the conference, and guest editors of this special issue, we want to thank our colleagues in the Centre for the Development of Academic Skills and other supporting departments at Royal Holloway for the assistance and hard work that a conference of this scale required. We are also grateful for the guidance of the EATAW board and the planning committee of the 2015 conference. Lisa Ganobscik-Williams and George Ttoouli are due our deep gratitude for their expert guidance, patient understanding and timely responses, despite the competing pressures and multiple responsibilities that both they and we have experienced. Many thanks go to all those who acted as reviewers, and of course to the contributors, who offered so many compelling and thought-provoking contributions and were responsive and timely throughout the review, revision and proofreading process.

The conference theme, 'Academic Writing Now: Pedagogy, Policy and Practice', was intended to generate contributions articulating a response to the shifting realities of Higher Education at the levels of policy, pedagogy and practice. The call for proposals was enthusiastically received, and the conference included 168 contributions in the form of 116 paper presentations, 8 symposia, 15 workshops, 20 poster presentations and 9 Lightning Talks. Perhaps not unsurprisingly, the themes most represented were pedagogy and practice, with some very insightful contributions on policy. Our three keynote speakers offered challenging perspectives on each of these three themes; their talks will be available on the EATAW 2017 website until autumn 2019, for those who wish to revisit them. ${ }^{1}$

\section{EATAW 2017 Keynote Speeches}

Prof. Rowena Murray launched the conference with the recognition of the expertise that our profession offers to the academy, and acknowledged the difficulties inherent in having a voice in policy. She posited the 'retreat' model that she and others have developed for academic writing as a possible means of disengaging from everyday activities to create space for policywriting. However, her problematisation of the various modes of disengagement that writers seek in order to prioritise writing not only articulated the scope of the challenge, but also identified a

\footnotetext{
${ }^{1}$ The keynote speaker videos are among 53 videos of sessions from EATAW 2017, hosted privately on YouTube so that they will be available in perpetuity. The entire playlist can be accessed here.
} 
systemic problem in academia that marginalises the importance of writing as legitimate, timeconsuming, essential work. This work, she argued, needs to be incorporated into the workloads of academics, including academic writing professionals. She called on us to create a community of practice that emphasises the sociality of writing and 'remutualises' it, and to reject the delegitimisation of scholarship as 'research-lite'. She closed by urging us to consider several areas of concern for policy-makers that are in pressing need of the voice of academic writing professionals.

On the second day of the conference, Prof. Ron Barnett's keynote picked up the issue of the peripherality of academic writing for academics. Drawing on his experience of releasing two to three million words of academic writing into the public domain, he reprised the critiques of the current state of academic writing as well as the current conditions under which we write. He provocatively suggested that he would ban the words 'explore' and 'discuss' from academic abstracts, the practice of hiding our own voices within compiled lists of citations, limited vocabularies and lack of creativity, and the use of scare quotes. He encouraged us as academic writing professionals to reflect on our own writing practices - to invest it with a great deal of care and to seek satisfaction in it. He posed the question, 'How many of us, when asked what we do, would respond with "I am a writer"?' Prof. Barnett's keynote stirred debate among conference attendees, some suggesting that perhaps he was 'preaching to the choir', others embracing its lucidity, elegance and clarity. As editors, reflecting once again upon his talk at the completion and publication of this special issue, we find new resonance in his exhortations. In the maieutic editorial process, we have come to understand anew the challenges of producing clear, compelling, original, engaging texts, and the multiple barriers to the honing and development of writing skills, including workloads, varied academic backgrounds, competing priorities, lack of institutional support, and the demands of writing itself. Prof. Barnett's keynote strikes us as a call to approach our writing with humility, academic writing professionals though we may be, to ask ourselves the same questions we might ask our students, and to strive to continue to develop our craft. We have learned an enormous amount through the process of hosting this conference and guest editing this special issue, and can highly recommend the experience to others.

Dr. Katrin Girgensohn's keynote on day three of the conference cut to the core of the institutional position of writing in the academy. She too tackles the thorny issue of institutional structure and policy, and proposes 'strategic action fields' and collaborative approaches to winning trust and legitimacy. Rather than reiterate Girgensohn's keynote address here, we invite you to read for yourselves her article, which grew out of her keynote address, and which has been published in this issue of JoAW. Indeed, we are grateful that she has developed this talk into an article, further summarised below, as it should enable us all to revisit and digest her thoughts, and perhaps work towards applying them to our own specific institutional contexts.

\section{Themes of the Special Issue}

The call for papers from conference contributors for this issue of the journal received significant interest, and we are pleased to present a diverse range of papers. The conference planning committee had introduced the genre of Lightning Talks - an informal but focused presentation of no more than five minutes - to the programme, and accordingly, this issue introduces the genre of Lightning Pieces to JoAW. These are short contributions of around 1,000 words outlining a practical writing intervention and a brief reflection on the questions this practice raises for ongoing research. We anticipated Lightning Pieces would attract contributions from those who gave Lightning Talks. In reality, two of the three Lightning Pieces that appear actually evolved from other genres - Alana James' workshop and David Hindley and Lisa Clughen's paper presentation. This poses interesting questions around perceived legitimacy, social capital and appetite for grappling with an unfamiliar genre, and we hope others might be interested in continuing to consider the affordances of the Lightning Piece genre.

We begin this special issue, however, with a contribution that was not presented at EATAW 2017, but rather which arose out of reflections on the event: Catalina Neculai's 'dialogue piece' encapsulates her observations and raises questions for the future direction of academic writing 
scholarship and, indeed, of policy, pedagogy and practice. Negotiating her own positionality as a critical insider, she raises vital questions about the field(s) inhabited by academic writing professionals, suggesting ways in which we might deploy our skills to examine and debate the position of our work within the political economy of the academy. As in the previous issue of this journal, we would like to invite readers to enter into dialogue with this piece, offering a counterpoint to its claims and a response to its invitation to imagine radical alternatives to the present model of academic writing instruction.

Responses to this piece will be published in a future general issue of JoAW. Please find further details in the Author Guidelines ('Dialogue Responses').

During the months of preparation of this special issue, many possible frameworks for presenting or thematising the articles have suggested themselves. We considered these groupings and the impact that the curation of texts may have on how they are read, even if a strictly 'linear' way of reading is less likely in an online journal, in which readers are probably 'dipping in' to pieces of interest rather than conceiving of the issue as a whole. We have arranged the pieces by 'approach' or by 'method' in the interest of promoting a 'systemic' view of the modes of scholarship within the field of academic writing, which might provoke further thought about the future directions of research in the field. Contributions were grouped into four categories: disciplinary reflections, scholarship of teaching and learning, localised research, and theorising academic writing.

\section{Disciplinary Reflections}

Notably, both pieces in the category of disciplinary reflections arise from research projects that involve interviews with academic writing professionals. Katrin Girgensohn's article, proceeding from her perceptive EATAW 2017 keynote talk, draws on her PhD research, which involved analysing interviews with leaders of writing centres via a grounded theory approach. She enters into dialogue with sociological literature on institutions and institutional work to understand the strategies leaders use to manage writing centres. Her piece advances a theoretical model for understanding the positionality of writing centres, which has important practical and policy implications for academic writing professionals.

Mary Davis raises important questions about the opportunity, or lack thereof, in the careers of academic writing professionals for academic research, writing and publication. She has interviewed a self-selecting group of writing instructors from various British universities to explore the value they place on publication and the barriers to scholarly activities that they experience. She articulates the value that publication holds for individuals in their professional careers, for institutions, and for the discipline, and calls for further research on these issues. The editors hope to begin this work by noting the primary modes in which the contributors to this issue (perhaps a non-representative but indicative sample of our field) have been able to pursue research agendas and publish their work.

\section{Scholarship of Teaching and Learning}

This leads to the second category of articles in this issue, pieces that represent the scholarship of teaching and learning (SoTL). These pieces arise out of real-world engagement with teaching and learning and incorporate the types of activities that are often an element of the ongoing development, monitoring, and evaluation of the effectiveness of learning activities: student feedback involving questionnaires or reflective responses, analysis of student marks, and in some instances, focus groups or interviews with students. Three of these five pieces are explicitly positioned as action research; three take place within multilingual HE contexts; and four engage with the question of the content / skills mix and pedagogical approaches to writing in the disciplines. Following Murray's keynote, and Girgensohn's and Davis's pieces above, this might suggest that academic writing professionals are necessarily pragmatic in their choice of methods for research publications. However, it may also indicate that they exploit their disciplinary resources in employing an inherently academic stance toward their daily teaching activities, and that they aim to create knowledge about teaching and learning that has a higher degree of rigour and is transferable beyond the immediate context. 
In the first article of this category, Yuan-Li Tiffany Chiu and Olga Rodriguez-Falcon use mixed methods, including focus groups and data analysis of student grades, to understand the impact of a social sciences transition module on student attainment. They propose the integration of academic literacies-based and genre-based pedagogical approaches as a platform for prioritising inclusion and the activation and integration of students' prior knowledge, indicating a dual ethical and pedagogical concern. It is worth noting that they press SoTL one step further by testing their reading of student performance, before and after the intervention, with focus groups and subsequent transcription and coding of recorded discussions, an analytical step which has yielded some particularly useful information about which strategies had impact on student learning.

In examining the re-design of an assignment on a Master's level Engineering course, Andreas Eriksson positions his call for increased collaboration between subject lecturers and academic writing professionals as an important practical intervention in English-Medium Instruction (EMI) contexts. He draws on analysis of texts produced in the course and interviews with students and the course manager, and presents the ways in which the course redesign facilitated alignment between content and writing specialists. The intervention resulted in students' improved mastery of disciplinary genre conventions.

Richard Hawley's analysis of an undergraduate skills module in a Classics department traces the stages of development and implementation, relying on student reflective writing from the module and feedback from colleagues and external examiners to understand the effects of the intervention. He emphasises the need to understand and engage with the diversity of resources that students bring to the learning context. He contextualises this in terms of the diversity arising at the crossroads of policy developments in both secondary and tertiary levels of education in the UK. Hawley presents an approach that embeds skills modules as a means of addressing diversity within programmes of study. We think this may invite further discussion of the value of embedding skills within content modules rather than in standalone skills modules.

Moving into the post-graduate context, José Brandão Carvalho, Luísa Álvares Pereira, and Rómina Mello Laranjeira draw on an exploratory study in the Portuguese context to contribute to the research of a large, collaborative, international study on students' approaches to the process of writing a dissertation, including the strategies they employ in completing their work, the problems they face, and what they feel they learn about both their subject and larger-scale writing projects. Carvalho et al. examine interviews with three Master's students within the Portuguese context in order to establish categories for further inquiry into the larger dataset. Their initial findings present the distinctive qualities of dissertation writing and its related developmental process. As a preliminary output from the larger study, representative of the type of SoTL achieved in local contexts, the article suggests questions about the scalability of this kind of research, and the challenge of designing and carrying out methodologies to obtain deep qualitative data from diverse contexts.

The final article in the SoTL category, by Melpomeni Ilkos, explores peer review in Greek institutions, where the practice has not yet taken hold, and examines educational culture as an explanatory tool, including student attitudes toward instructor authority and the value of peer input. She uses student writing produced in an English-medium undergraduate academic writing module as a source of data, cross-referenced with the results of an online questionnaire that includes demographics, previous experience with peer review, and self-assessed level of English language competence. Her efforts yield new insights into the role of gender and anonymity in peer review, and her examination of the reinforcement of the comments of peer reviewers by markers suggests additionally useful considerations for peer review pedagogy.

\section{Localised Research}

A great strength of the field of academic writing is the localised nature of much of the scholarship. As Rowena Murray pointed out, this could be (and has been) maligned as notquite-research. Its prominence may be a function of the inescapable reality faced by practitioners working on non-academic contracts who conduct research 'on the job', but we think this localised, grassroots, often praxis-oriented tradition of enquiry is both valuable and somewhat unique to the field. For not only does local research in writing feed directly back to 
local practices, but it is also the case that such research, far from being parochial and hard to apply to other contexts, brings wider pedagogical and political realities to the surface. Furthermore, our contributors in this section have scaled their scholarship project into robust academic investigations. We might call it 'glocal' research. In this vein, Deryn P. Verity's (local, US-based) analysis of tutor reflective narratives on an MA TESL (Teaching English as a Second Language) programme reveals the highly iterative, transformative nature of tutor development. Using the concept of prolepsis as a heuristic, Verity uncovers examples of tutors looking forwards (to an expert future self) and backwards in their reflexive practice as important indicators of development. The article is itself an instance of prolepsis, with Verity's political call to make tutor education a more professionalised and established facet of teacher education.

The 'under-professionalised' nature of much tutor development is also the theme of Elizabeth Caldwell, Katharine Stapleford, and Amanda Tinker's article, which reports on findings from a conversation analysis of writing tutorials in another locality, a northern English university. As well as providing insights into the world of the writing tutorial, the authors draw unconventional comparisons with other 1:1 encounters, such as the telephone helpline and psychotherapy sessions. Their findings show the writing tutorial as a kind of genre, or proto-genre, with a set of clear and predictable phases through which typical tutorials pass. In so doing, the authors exemplify good practice in elicitation techniques that have clear pedagogical applications in the form of tutor development materials. These materials, which form a key follow-up project planned by the authors, should contribute to making Verity's proleptic call to bring tutor development from the margins of teacher education a reality.

Colleagues tasked with the marking and evaluation of large amounts of student work will doubtless recognise the sentiment behind the title of Sarah S. Haas' article, which is a fine example of 'local-to-global' research. The aim of the project, initially focused on her classroom practice, was to answer the deceptively simple question of what causes readers to engage or disengage with a piece of student writing. In Haas' case it was the anxiety and trepidation of marking a large number of student papers ('The Pile'), and why, specifically, so many of the papers elicited disengagement on the part of the reader. Haas' investigation, beginning with her own self-analysis of (dis)engagement with her students' papers, grew to include the insights from teaching staff and students themselves. The result - a highly original 'Reader Engagement Framework' - has wide pedagogical applications. It will be interesting to see how this framework finds application in different contexts; one interesting perspective, particularly in view of the multilingual reality of much academic writing, is the question of reader-versus writer-responsive writing, that is, the extent to which it is the writer's or reader's responsibility to achieve clarity (e.g., Mauranen 1993). It will be fascinating to see if cultural-rhetorical differences manifest themselves in how the Framework is utilised.

This trans/multilingual reality of academic writing at the international academy, and the differing reader expectations that may ensue, is the theme of Melanie Brinkschulte, Ella Grieshammer, and Monica Elena Stoian's article, which outlines a translingual pedagogical approach to academic writing, developed at the University of Göttingen, Germany. The authors' starting-point is the fact that in Germany (and elsewhere) academic discourse segues from German at an undergraduate level to English at postgraduate level, reflecting the dominance of English in academia. Leaving aside the rights and wrongs of English as the linguistic hegemon in academic research, a process which John Swales memorably referred to as 'English as Tyrannosaurus rex' (1997: 373), the Anglophone academic world is currently an inescapable reality. The authors' pedagogic approach, however, quite rightly recognises multilingualism as a 'chance', as they put it, and their pedagogy demonstrates how students' languages are best conceptualised as resources that can be brought to bear on the process of producing (English) academic prose. Utilising insights gained from the strategies of multilingual scholars obliged to publish in English, the authors identify a welter of 'translingual' writing strategies, including drafting and peer reviewing, which can be employed by student-writers tasked with producing academic writing in English. It is an irony that will not be lost on many readers that the authors' paper enacts the very issue it raises.

Self-assessment: theorised, pedagogically sound, the weapon-of-choice for any would-be promoter of the progressive, learner-centred writing classroom. And yet it is a method often 
maligned by the learners themselves, as it seems to collapse often deeply-held perceptions of traditional expert-novice relationships. Katharina Weiss explores this tension, as well as the crucial problem of just how reliable, accurate and effective student self-assessment is, in a local and, once again, translingual context: an English Medium Institution in Greece. Weiss' paper interrogates the challenges of achieving objective measurement, and taps usefully, we think, into the merits or otherwise of the growing appetite in Higher Education for metric-based accountability regimes.

\section{Theorising Academic Writing}

If academic writing is - as Prof. Dr Wolfgang Drechsler put it at the 2015 EATAW conference in Tallinn, Estonia - 'the meta-discipline' (Event Media Estonia 2015) - and it is a claim with which philosophers (and possibly physicists) may take considerable umbrage - then part of academic writing's remit must surely be to interrogate its own practices. We see this reflected in the several contributions to this issue which seem to enact the very issues they explore. Such epistemological introspection is an important facet of the scholarship of academic writing. We have seen over the years many attempts to problematise and challenge the conventions and epistemological foundations of academic writing, most notably in Academic Literacies-inspired research (e.g., English 2011, Lea and Street 1998, Lillis 2001). While hugely valuable, there is a slightly disheartening (or gratifying, depending on one's point of view) irony in the realisation that this research seldom if ever manages to break out of the very academic conventions and epistemologies it critiques. Theresa Lillis (2001) comes closest in her influential book Student Writing: Access, Regulation, Desire, which, although conforming largely to the conventions expected of an academic monograph, occasionally offers intriguing mini-sections, called 'connections', which invite the reader to share glimpses of Lillis' autobiographical, writerly self as an epistemological imperative. But overall, this intellectual critique has not managed to dismantle the genre of academic discourse to any great degree.

Yet Tom Muir's paper makes a bold attempt to do just this in a playful yet deadly serious, genre-bending exploration of risk and the occult in academic writing. That his submission was a gamble is clear from the fears he expresses at daring to include ('I cannot pretend it doesn't make me anxious') discussion about, of all things, the lost nexus of science and magic. But we think readers will find in Muir a captivating explorer, whose ideas branch out, like the page from Nick Sousanis' Unflattening (2015) reproduced in the article, into the worlds of the other contributions to this issue. We must at this point make the following editorial confession, as it provides an example of the issues with which Muir grapples: that, in order to mitigate the risk of readers baulking at the academic conventions flouted, we requested the addition of the 'discourse on method' box accompanying the introduction. In retrospect, perhaps we should have demurred from such editorial meddling, and published it sans caveats, as a 'writerly' piece about which readers can form their own judgements, without, in Muir's words, 'metadiscoursally signal[ling] what I am up to'. And we did consider removing it, but then realised that its presence had become an ineluctable part of the textual history of the article, an example, perhaps, of the very kind of academic reaction to unconvention that, Muir argues, following Mary Scott (2014), renders the 'risky' text invisible or 'ghostly'. So the 'discourse on method' box stays. Muir ends with a call - a pedagogic one - for students to be aware of the affordances of the unacademic, not least as a way of addressing the often vexed issue of student 'voice'.

Awareness, or self-awareness, seems to be the lodestar for Bonnie Devet's reflections on how learning transfer theory can be brought usefully to bear on tutor development. In a review of the scholarly terrain of learning transfer, Devet identifies six analytical 'lenses' through which transfer may be rendered visible to both tutor and student. These six categories - content, context, genres, prior knowledge, reflection and disposition - frame examples of good tutoring practice from Devet's own writing centre context. So, for example, we see how reflective discussion allowed one biology student to gain awareness of different writing genres; how utilising a combination of categories led one tutor to turn their student's confusion about differences between the book review and essay genres into an opportunity for transfer - with powerful results. What these lenses provide is a usable heuristic for tutor training: a developmental framework which allows tutors to reflect critically on their own practices. 
Amanda French takes up an issue readers will undoubtedly recognise: that of academic writing as a profoundly emotional undertaking. French argues that the physicality and emotions experienced by students navigating the treacherous straits of academic writing are routinely underplayed by subject lecturers, who still, for French, cling limpet-like to long discredited notions of academic writing as unproblematic autonomous skill. She argues rather for a more holistic approach to academic writing, one that appreciates the centrality of the affective domain in student writing. Concretely, it involves the opening up of 'safe' spaces to develop writing, via unassessed writing tasks such as blogs, collaborative writing, and reflective journals. We think that this is perhaps (and it would be interesting for 'subject lecturers' to read this piece) starting to happen in some cases; students in many degree programmes (at least in the UK) are writing in more diverse genres. Certainly, the reflective journal is fairly common, and students are increasingly expected to write blogs (see Hindley and Clughen below), and other texts for different audiences. The problem, it seems to us, is that much of this is still subject to formal assessment (so continues to deny the safe environment French's paper quite rightly emphasises), and is often presented to students with little guidance as to the genre expectations. Diversify the genres too much, one might contend, and the result, as the English proverb puts it, is to make students jacks of all trades, masters of none.

\section{Lightning Pieces}

As little lenses on learning interventions, the three Lightning Pieces focus on the importance of having different forms of writing in a central position in the curriculum. Alana James, reporting on an intervention at the host institution of EATAW 2017, Royal Holloway, presents the development of an academic skills module for Psychology. The module evolved over three years from lectures delivered solely by psychology subject lecturers to one which involved codelivery with Careers and Library, and ultimately, with academic skills staff. This allowed the utilisation of specialists and hence a more nuanced and better-mapped module. While students were satisfied by this intervention, as James acknowledges, performance impact was not measured; it will be interesting to see if performance tracking is undertaken in future and, further, whether there is a discernible impact on students' learning gains as a result, though we recognise all too well the perils, both methodological and ethical, that underlie this increased desire to measure.

The 'essay' as prototypical student genre is well known, and its centrality increasingly questioned. How relevant is the genre in the fast-moving, social media-obsessed, cut-andpaste generation of modern university students? Will Self, English novelist and Professor at Brunel University, UK, has famously binned the 'essay' altogether in his courses, arguing it to be an obsolete genre (Self and Watkin 2016). David Hindley and Lisa Clughen argue, in their Lightning Piece, that blogging offers a more inclusive and potentially transformative academic activity that may help students to develop their academic identities and voices in ways the traditional essay cannot. Echoing Amanda French's call to acknowledge the emotional, Hindley and Clughen premise the paper on the 'deep anxieties' felt by many students faced with producing good academic work. Blogging, the authors argue, 'opens possibilities for students to author their scripts rather than remain alienated from them'. We feel sure Will Self would heartily agree.

Justin O'Brien extols the virtues of brevity and concision in a provocative, polemical piece critiquing the default-setting in many institutions of the 'long-form' essay ('not another 2,000word essay'). O'Brien argues for a minimalist approach to assessment strategy that is inspired by advertising's preference for the 'power of the short', the absence of redundancy, and absolute clarity of message. He presents several practical suggestions for 'short' assignments, including the précis, five-minute podcasts and presentation slides. Whilst we agree that academia is often bedevilled by a 'systematic inertia' that keeps things conservative, and that 'production-led thinking stymies [...] innovative practice', we do not think the essay is as yet an endangered species. Colleagues may wish to reflect on the prevalence of certain academic genres in their own contexts and, crucially, whether the diversification of genres is likely, desirable, or even teachable. Not least, there is the question of whether more diverse writing 
regimes reflect positively on essay-writing competence, a question we invite readers to consider.

\section{Conclusion}

The process of editing this special issue of JoAW has been what can only be described as a labour of love. We ourselves have experienced many of the challenges familiar to academic writing practitioners, scholars and researchers, particularly those without academic tenure and/or a research remit. Carving out sufficient time and headspace to do justice to the submissions we received has been at times difficult, but worth it. The quality of work submitted for consideration was humbling, and it was with a heavy heart that we were not able to include some of the submissions in this issue. But watch this space: some submissions have been earmarked for future issues of JoAW, so the spirit of EATAW 2017 endures a little longer.

Furthermore, we hope that, as preparations are underway for proposals for EATAW 2019, the momentum will build from these proceedings from EATAW 2017. We suggest that this consideration of the predominant research methods in our field might reveal gaps in the body of research, and consideration of the kinds of knowledge we are creating about writing and about our field. We hope the contributions presented here will inform and inspire ongoing research and scholarship, as well as further understanding of our field and its discourses, practices and pedagogies - and perhaps even provide resolve, strategies and insights to influence and shape our policy contexts.

To close, we once again invite readers to respond to Catalina Neculai's Dialogue. Catalina raises fascinating - and difficult - questions about the future of academic writing, not only in terms of scholarly activity but also its positioning (political and pedagogic) within the modern academy. We would particularly welcome responses that offer radical, disruptive solutions that challenge the status quo by questioning 'common sense' assumptions about the trajectory and function of academic writing teaching and scholarship.

Laryssa Whittaker

Royal Holloway, University of London

Stuart Wrigley

Royal Holloway, University of London 


\section{References}

EATAW 2017 (2016) EATAW 2017 - 9th conference of the European Association for Teaching Academic Writing. [online] available from <http://eataw2017.org/> [13 November 2018]

Event Media Estonia (2015) EATAW 2015: Opening and Keynote Speech by Dr Ulla Connor. [online] available from <https://www.youtube.com/watch?v=jeLzye6C_Xg> [14 November 2018]

English, F. (2011) Student Writing and Genre. London: Continuum

Lea, M., and Street, B. (1998) 'Student Writing in Higher Education: An Academic Literacies Approach'. Studies in Higher Education 23 (2), 157-172

Lillis, T. (2001) Student Writing: Access, Regulation, Desire. London: Routledge

Mauranen, A. (1993) 'Contrastive ESP Rhetoric: Metatext in Finnish-English Economics Texts'. English for Specific Purposes 12, 3-22

Scott, M. (2014) 'Error" or Ghost Text? Reading, Ethnopoetics and Knowledge Making'. In Risk in Academic Writing: Postgraduate Students, Their Teachers and the Making of Knowledge. ed. by Thesen, L., and Cooper, L. Bristol: Multilingual Matters, 203-218

Self, W., and Watkin, W. (2016) 'How a course about violence changed the way students are taught and assessed'. Times Higher Education [online] 14 July. available from $<$ https://www.timeshighereducation.com/features/how-a-course-about-violencechanged-the-way-students-are-taught-and-assessed> [28 November 2018]

Sousanis, N. (2015) Unflattening. Cambridge, MA: Harvard University Press

Swales, J. (1997) 'English as Tyrannosaurus rex’. World Englishes 16, 373-382 